\title{
Mikroskopische Untersuchungen über die chronische plastische Dermatitis.
}

\author{
Von \\ Dr. Louis Heitzmann, \\ Arzt am Deutschen Dispensary in New-York. \\ (Mit 5 Figuren.)
}

In meiner Abhandlung über acute Dermatitis habe ich wiederholt auf die Verbindungen der Entzündungskörper vermittelst feiner, fädchentörmiger Fortsätze aufmerksam gemacht. Diese Fortsätze sind ausserordentlich zart, was wohl die Thatsache erklärt, dass sie bisher nur von wenigen Mikroskopikern, gesehen wurden. An in Canada-Balsam montirten Präparaten wird man sie nicht seben können. In Glycerin aufbewahrte, vorher mittelst Citronsäure gehärtete Präparate liıssen diese Fortsätze auch nur bei stärkeren Vergrösserungen (800 bis 1000 linear) deutlich erkennen. Die bei 500 und 600fachen Vergrösserungen gezeichneten Figuren zeigen nur undeutliche Verbindungen; betrachtet man aber diese Figuren mit einer Lupe, so wird man die in Rede stehenden Verbindungen schon viel klarer erkennen können.

Es genügte nicht, von einer Verbindung der Entzündungskörperchen untereinander mittelst eines schmalen Saumes einer interstitiellen Kittsubstanz zu sprechen; letztere ist ein secundäres Product und als solches nicht mit Lebenseigenschaften ausgestattet. Erst wenn man die, die Kittsubstanz senkrecht durchziehenden konischen Fädchen erkennt, Bildungen der eigentlichen lebenden Materie, kann man von einer lebendigen Verbindung sämmtlicher Entzündungskörperchen sprechen. Dann wird aber auch der Aus- 
spruch klar, dass ein entzündetes Gewebe, in unserem Falle die Lederhaut, ein Gewebe bleibt und sobald die acuten Entzündungserscheinungen aufgehört haben, neues, mit Grundsubstanz ausgestattetes Gewebe zu erzeugen vermag.

Im Verlaufe der acuten Entzündung ist, wahrscheinlich in Folge des vermehrten Zuflusses von Nährmaterial, neue lebende Materie entstanden. Diese erscheint zunächst in Gestalt von Entzündungskörperchen. Wir brauchen eben nur die Reihenfolge der Erscheinungen, welche zum Auftreten von Entzündungskörperchen geführt haben, umzukehren, und wir verstehen sofort die Bildung neuen fibrösen Bindegewebes. Die ursprünglich soliden Klümpchen der lebenden Materie werden zunächst vacuolirt und treten hierauf in den Zustand reticulirten Protoplasmas, wobei in jedem Klümpchen ein centraler, homogener, vacuolirter oder reticulirter Kern zurückbleibt. Je weiter die Umbildung zu Protoplasma vorschreitet, desto mehr verliert der Kern sein compactes Aussehen, desto mehr nimmt derselbe eine bläschenförmige Gestalt an und im Stadium der Indifferenz, das heisst, wenn das Protoplasma sich der Infiltration mit Grundsubstanz nähert, sind die Kerne nur noch wenig deutlich ausgesprochen. Die neu entstandenen Protoplasmakörper zeigen mannigfache Gestalten; sie sind globulär oder oblong oder spindelförmig, bäufig in ihren Contouren abgeplattet. Dadurch entsteht das Aussehen von Endothelien, welche von einander durch schmale Leistchen von Kittsubstanz getrennt, jedoch unter einander durch feine, die Kittsubstanz durchziehende Fädchen lebender Materie verbunden sind. Derartiges Protoplasma kann unmittelbar zu Grundsubstanz umgewandelt werden, oder das Protoplasma zersplittert zu schmalen Spindeln, welche der Sitz einer Infiltration mit Grundsubstanz werden. In ersterem Falle haben wir breite Bündel fibrösen Bindegewebes als Resultat ror uns, in welchem das streifige Aussehen vollständig fehlt; in letzterem schmale Bündel von ausgesprochen fibrillärem Bau. Der Vorgang ist genau derselbe, wie ich ihn in der normalen Entwicklung des Derma ${ }^{\mathbf{1}}$ ) geschildert habe. Das Endresultat ist nur darin verschieden, dass in Folge der im acuten Stadium mehr oder weniger ver-

I) Dieses Archiv 1890. p. 631. 
Mikroskop. Untersuchung. über die chronische plastische Dermatitis. 691

mehrten Bildung lebender Materie ein Gewebe entsteht, welches an Umfang mehr oder weniger zugenommen hat. Diesen Zustand bezeichnen wir nach Virchow als Hyperplasie des Bindegewebes.

Nicht die Entzündung selbst ist chronisch geworden, sondern deren Resultat, die Hyperplasie. Begreiflicher Weise kann ein in Folge von acuter Entzündung hyperplastisch gewordenes Bindegewebe am Wege der sogenannten Resorption in den Normalzustand zurücktreten, wobei stets das Zwischenstadium der Rückkehr zu indifferentem Protoplasma durchgeführt wird. Nicht selten geschicht es jedoch, dass schon hyperplastisch gewordenes Bindegewebe der Sitz neuer Nachschübe von acuter Entzündung wird, sogenannte subacute Entzündung, wobei in jedem Nachschube eine gewisse Menge lebender Materie neugebildet wird und schliesslich ganz beträchtliche Verdickungen der Lederhaut entsteben.

Dem Kliniker sind die Bilder der chronischen Hyperplasie des Derma ganz geläufig, insbesondere bei chronischem Eczem, bei Prurigo, Dermatitis papillaris, Pachydermie und Llephantiasis Arabum. Neuerdings haben wir auch im Myxoedem einen Krankheitsprocess kennen gelcrut, bei welchem eine beträchtliche Noubildung von Gewebe dor Iolerhaut entsteht. Bei Kiczem und bei Prurigo sind es augenscheinlich die durch das Kratzen exzeugten mechanischon Insulte der Haut, welche zur Hyperplasie des Derma führen. Bei Dermatitis papillaris ist es die durch einen lange andaueraden Eiterungsprocess hervorgerufene Reizung des Derma, vorwiegend in dessen oberen Lagen und des Papillarkörpers. Aehuliche Ursachen, nämlich chronische Eiterungs- und Verschwärungsprocesse führen auch zur Pachydermie, zumal an den Unterschenkeln. Bei der Elephantiasis wird eine subacute Lymphangoitis als Ursache angesehen. Bei Myxoedem ist die Ursache der Hyperplasie des Derma nicht bekannt und steht mit der Schilddriuse, oder vielmehr deren Mangel, in irgend einem, wenn auch vorläufig noch räthselhaften Zusammenhange. Betrachten wir uns bei schwacher Vergrösserung das Bild einer stark verdickten Lederhaut, als Folge von wiederholten subacuten Nachschüben bei chronischem Eczem. (Siehe Figur 1.) 


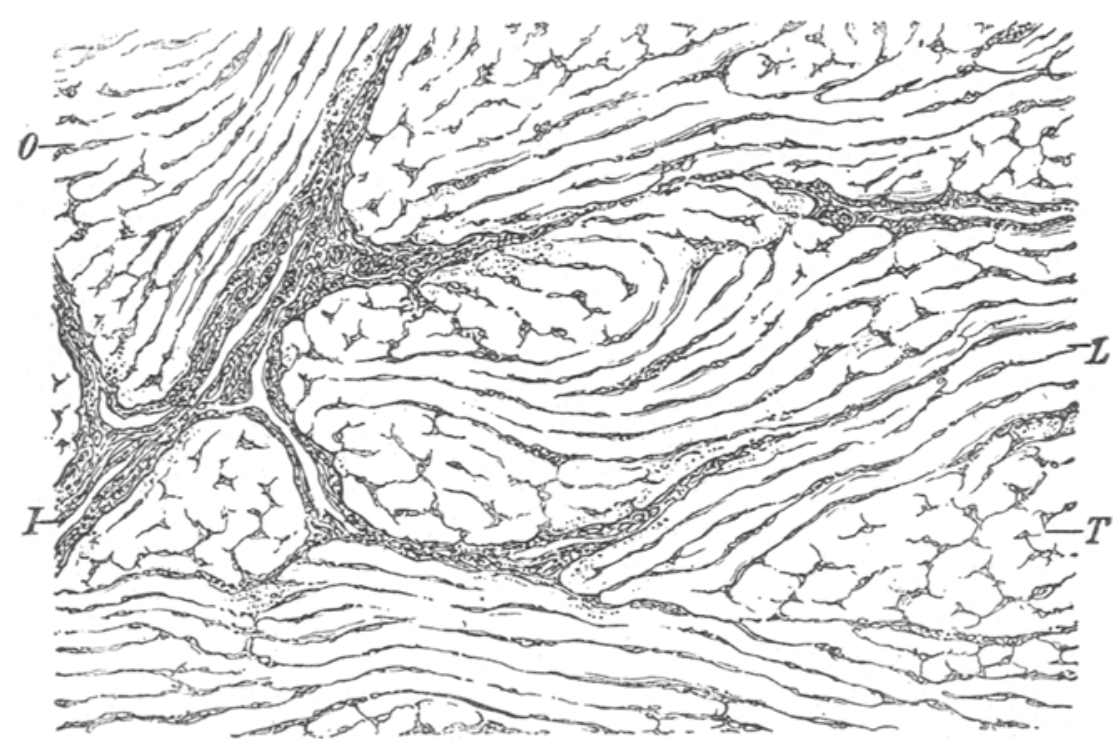

Fig. 1. Chronischehyperplastische Dermatitis. Vergrösserung loofach. $I=$ Interstitien erster Ordnung, zahlreiche Entzündungskörperchen und Gruppen dunkelbraunen Pigments enthaltend. $L=$ Bündel von dichtem fibrösen Bindegewebe im Längsschnitt. $O=$ Bündel von dichtem fibrösen Bindegewebe im Schiefschnitt. $T=$ Bündel von dichtem fibrösen Bindegewebe im Querschnitt.

Wir erkennen Bindegewebsbündel in Längs-, Quer- und Schiefschnitten. Zwischen den Bündelgruppen sehen wil Felder erfüllt mit indifferenten Protoplasmakörpern und zart fibrillärem Bindegewebe, Blutgefässe in wechselnder Menge führend. Wir erkennen hier die Interstitien erster Ordnung, wie dieselben auch dem normalen Derma zukommen, und die unmittelbar in die Interstitien zweiter Ordnung übergehen. Die Anzahl beider ist grösser als im normalen Derma. Die Interstitien dritter und vierter Ordnung sind am Vieles mehr ausgeprägt, als im normalen Zustande, dadurch bedingt, dass die Menge der interstitiellen Protoplasmas beträchtlich vermehrt ist. Aber auch die Bündel selbst sind um ein Gutes breiter als im normalen Zustande und überdies dadurch ausgezeichnet, dass sie stark lichtbrechend und nahezu homogen erscheinen. In den oberen Lagen des Derma begegnen wir auch streifigen Bündeln; jedoch 
ist die Menge derselben merklich geringer als jene des homogenen. Elastische Fasern sind in unseren Präparaten nur in geringer Menge enthalten. Der Papillarkörper ist ebenfalls vergrössert; die Papillen sind verlängert und verbreitert, gleichfalls aus derberen Bündeln aufgebaut, als normal. Blutgefässe sind in merklich geringerer Anzahl vorhanden, als im normalen Derma und dessen Papillarkörper. Das Rete mucosum ist verbreitert, tief braun pigmentirt, insbesondere das Stratum granulosum nahezu schwarz. Ein eigentliches Stratum lucidum fehlt. Die Epidermalschicht ist verbreitert und gleichfalls strecken. weise braun pigmentirt.

Studiren wir nun die byperplastische Lederhaut bei starker Vergrösserung. (Siehe Fig. 2.)

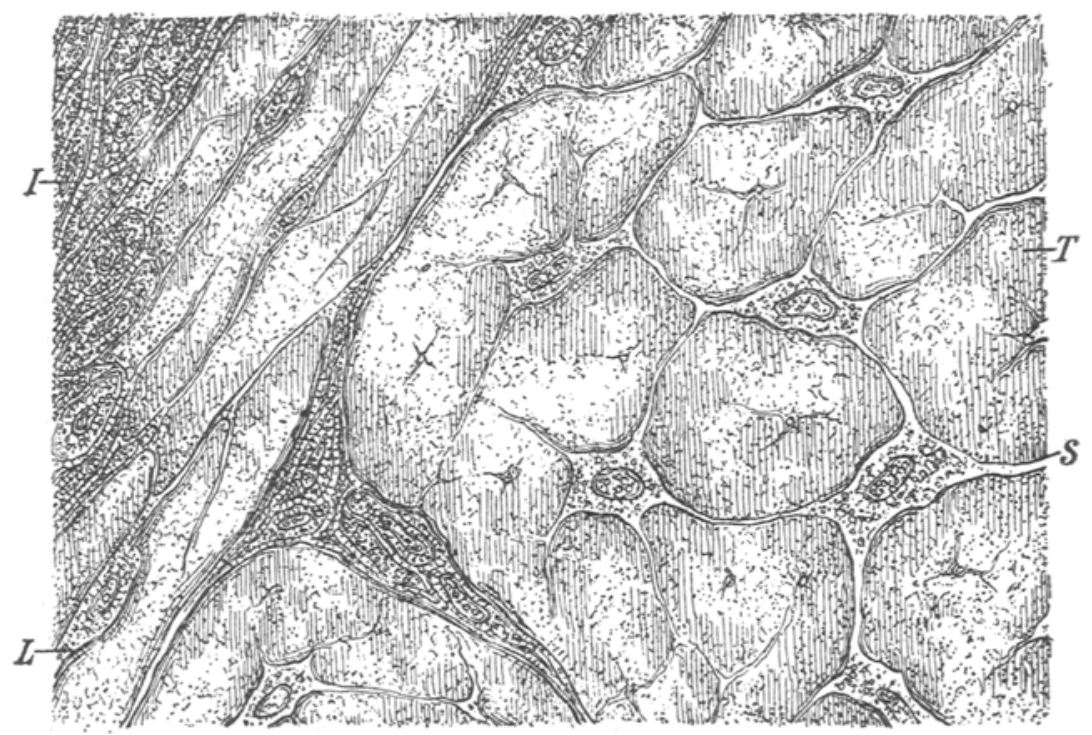

Fig. 2. Chronischehyperplastische Dermatitis. Vergrösserung 1000fach. $I=$ Interstitien zweiter Ordnung mit Entzündungskörperchen gefüllt. $L=$ Bündel eines dichten fibrösen Bindegewebes im Längsschnitt. $T=$ Bündel eines dichten fibrösen Bindegewebes im Querschnitt. $S=$ Interstitien zwischen den Bündeln, mit Protoplasma gefüllt, ein sternförmiges Netz erzeugend.

Wir exkennen in unserer Abbildung Insterstitien zweiter, dritter und vierter Ordnung; diejenigen der dritten Ordnung 
sind noch gut ausgeprägt und mittelst protoplasmatischer Fortsätze untereinander verbunden. In Folge der starken Lichtbrechung der Bündel erscheinen die Fortsätze nur als helle Ausläufer, in welchen die Structur des Protoplasmas nicht zu erkennen ist. Die Interstitien vierter Ordnung sind in Querschnitten von Bündeln deutlich erkennbar; sie enthalten drehrunde Körper, welche auf eine beginnende Bildung von elastischer Substanz hinweisen. Der Zusammenhang dieser Interstitien mit jenen der dritten Ordnung lässt sich nicht verfolgen. Ueber den Ursprung dieses derben hyperplastischen Gewebes geben uns jene Strecken Aufschluss, welche sich noch im Stadium der protoplasmatischen Indifferenz befinden (Fig. $2 d$ ). Hier sehen wir die vorhin beschriebenen, nahezu endothelialen Protoplasmakörper, theils kernlos, theils mit blassen Kernen versehen und untereinander sehr deutlich mittelst feiner Fädchen verbunden. Hart an diese Bildungen stossen polygonale Felder, in welchen die Protoplasmastructur theilweise noch erkennbar ist, während die Hauptmasse sich im Zustande der Infiltration mit Grundsubstanz befindet. Derlei Bilder weisen mit Bestimmtheit darauf hin, dass auch im neugebildeten Derma die Felder der Grundsubstanz aus Protoplasma hervorgehen und die Infiltration mit Grundsubstanz nur die Maschen des Netzes der lebenden Materie betrifft, während das Netz der letzteren zwar unsichtbar geworden, aber dennoch vorhanden ist, worauf die, allerdings nur blasse Körnung der Grundsubstanz hinweist.

Schon mittelstarke Vergrösserungen genügen, um auch im Papillarkörper das Wesen der Hyperplasie erkennen zu lassen. (Siehe Fig. 3.)

Die Structur ist dieselbe, wie im normalen Papillarkörper eines Individuums mittleren Alters. Der Unterschied besteht darin, dass sämmtliche Bündel verbreitert und zwischen denselben die Protoplasmakörper vermehrt sind. Jene Protoplasmakörper, welche zur Bildung von Bündeln in den Papillen geführt haben, waren wahrscheinlich spindelförmig, entsprechend den unregelmässigen Spindelformen der zwischen den Bündeln enthaltenen, freien Protoplasmakörper selbst. Die Capillaren dieses Gewebes sind vielfach in ihrem Durchmesser verbreitert, und zeichnen sich durch gestreckten Verlauf aus. 


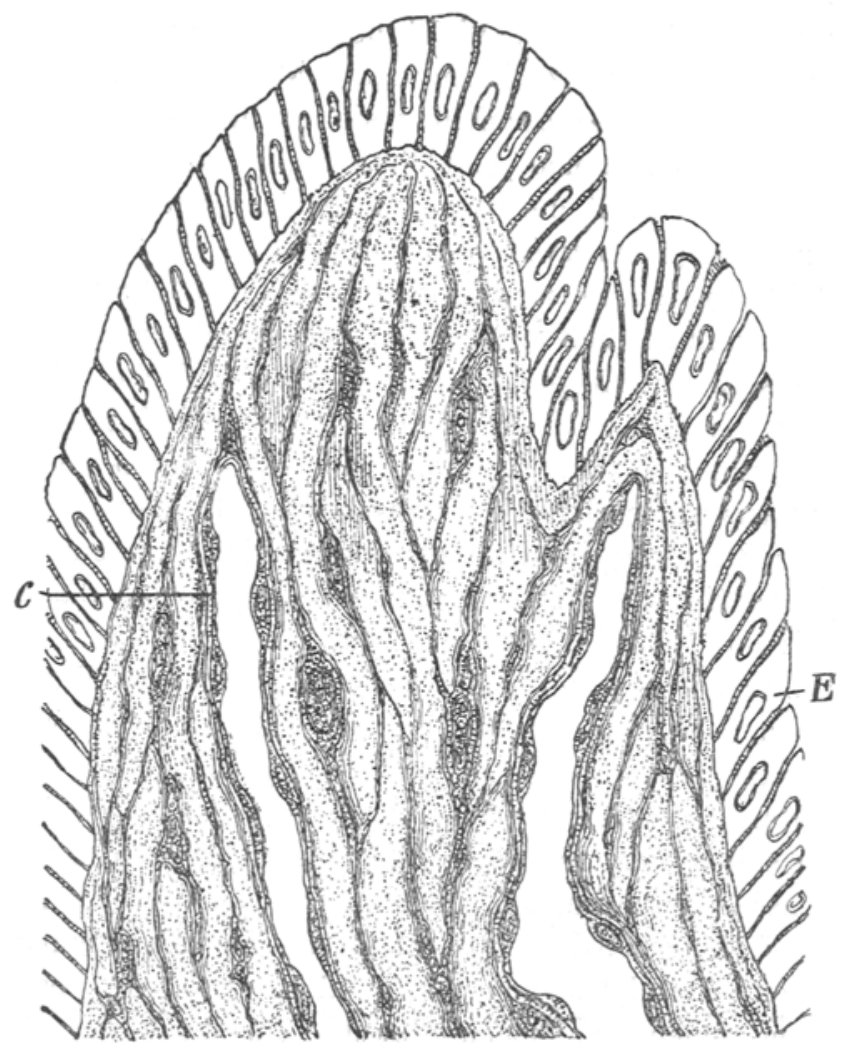

Fig. 3. Papille von einer hyperplastischen Lederhaut. Vergrösserung 600fach. $E \doteq$ Säulenepithel. $C=$ Capillarrohr.

Die Hyperplasie des Derma ist insbesondere in der sogenannten Pachydermie und Elephantiasis Arabum ausgeprägt und habe ich mehrere Fälle dieser Krankheiten, welche theils an den äusseren Geschlechtstheilen, theils an den unteren Extremitäten aufgetreten waren, mikroskopisch untersucht.

Im Sinne Virchow's ${ }^{1}$ ) sollten wir die Bezeichnung „Pachydermie" auf die Hyperplasie der oberen Lagen des Derma und des Papillarkörpers beschränken; während als „Elephantiasis Arabum " die durch wiederholte Lymphgefässentzündung veran-

I) Die krankhaften Geschwülste. Band I. 1863. 
lasste Hyperplasie des gesammten Derma, des Unterhautgewebes und eventuell sämmtlicher Weichtheile einer Extremität zu bezeichnen wäre. Letztere Form zählt Virchow zu den Geschwulstbildungen und bezeichnet sie als diffuse Fibrombildung, für welche er den Namen "elephantiatische" vorschlägt. BirchHirschfeld ${ }^{1}$ ) zählt die Elephantiasis im Gegensatze zu Virchow zu den productiven Entzündungen.

Bei Pachydermie ist wohl stets das Epithellager beträchtlich verbreitert, und da auch die Papillen einer Vergrösserung in allen Durchmessern unterliegen, erscheint die Oberfläche schon dem freien Auge höckerig, drusig oder selbst warzenförmig (Pachydermia verrucosa), In den von mir untersuchten Fällen von Elephantiasis war weder die Vergrösserung des Papillarkörpers, noch eine Zunahme des Epithels nachweisbar. In einem Falle von Elephantiasis der grossen Schamlippe erschien das Epithel sogar bis auf wenige Lagen verdünnt.

Ueber die Herkunft des verdickten Bindegewebes gehen heute noch die Ansichten auseinander. Kaposi ${ }^{2}$ ) spricht von einem entzündlichen oder lymphatischen Oedem, in welchem reichlich Lymph- oder farblose Blutkörperchen suspendirt sind. Letztere sollen sich verlängern, zu Fasern ausziehen und dadurch, dass sie untereinander verschmelzen, ein Gewebe erzeugen. $\mathrm{Ziegler}{ }^{3}$ ) hingegen leugnet mit Recht die Betheiligung eines plastischen Exsudates an der entzündlichen Gewebsbildung Nach ihm gehen die neuen Gewebe am Wege der directen oder indirecten Zelltheilung aus den früheren Gewebszellen hervor.

Bei meinen eigenen Untersuchungen habe ich, entsprechend einer klinisch schon längst bekannten Thatsache, zwei Hauptformen kennen gelernt, nämlich die weiche oder gallertartige, Elephantiasis mollis, und die harte oder fibröse, Elephantiasis dura. In der ersteren Form besteht die hyperplastische Cutis aus myxomatösem oder myxofibrösem, in der letzteren aus feinbündoligem fibrösem Gewebe.

1) Lehrbuch der pathologischen Anatomie. Bd. 1, 4. Auflage 1889.

2) Pathologie und Therapie der Hautkrankheiten. 2. Auflage 1883.

3) Lehrbuch der allgemeinen und speciellen pathologischen Anatomie. Bd. I, 6. Auflage 1890. 
Mikroskop. Untersuchung. über die chronisohe plastische Dermatitis. 697

Untersuchen wir zuerst ein Präparat von Elephantiasis mollis der grossen Schamlippe. (Siehe Fig. 4.)

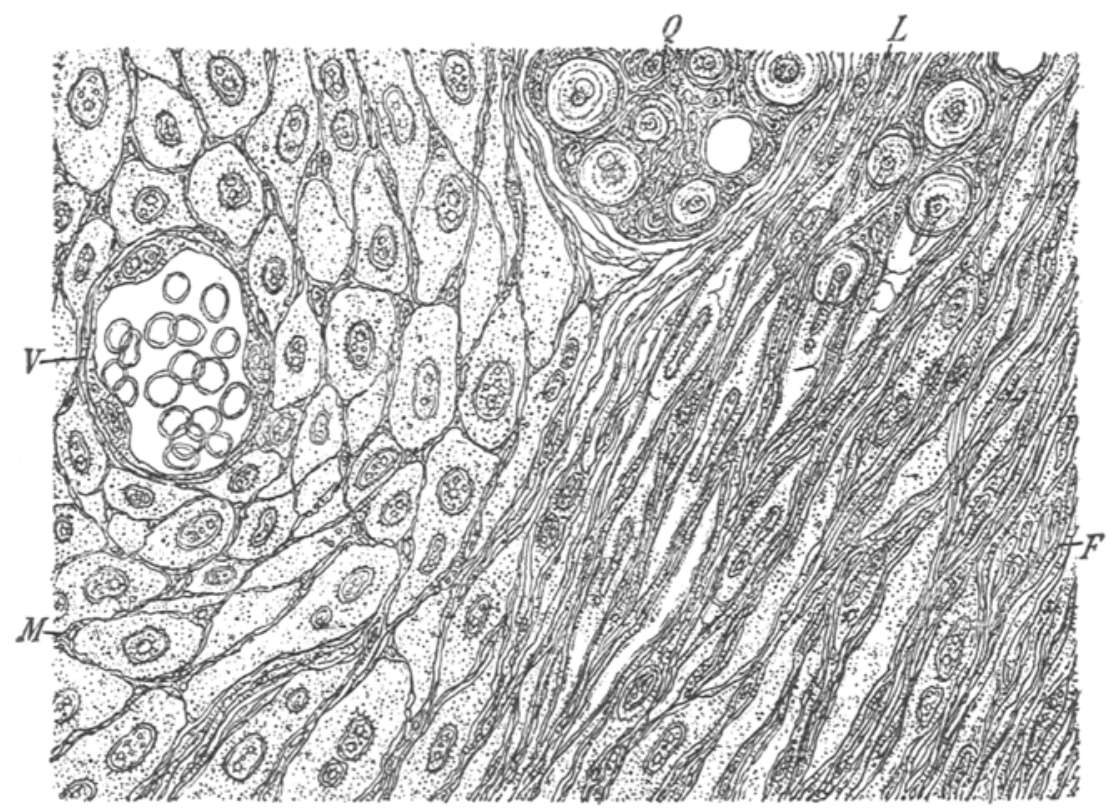

Fig. 4. Chronische Hyperplasie. Elephantiasis mollis der grossen Schamlippe. Vergröserung 500fach. $M=$ Myxomatöses Gewebe. $F=$ Myxo-fibröses Gewebe. $L=$ Längs- und Schiefschnitte von glatten Muskelspindeln. $Q=$ Querschnitte von glatten Muskelspindeln. $V=$ Vene oder grosses Capillar im Querschnitte.

Feine Schnitte erscheinen dem unbewaffneten Auge honigwabenartig durchbrochen, die Oberfläche nahezu glatt. Schon bei schwacher Vergrösserung erkennt man, dass das bedeckende Epithellager bis auf drei oder vier Schichten cubischer Epithelien reducirt ist, und zahlreiche Verlängerungen in das Hauptgewebe sendet, welche möglicherweise den früheren Thälern zwischen den Papillen entsprechen. Das Gewebe ist vorwiegend myxomatös in den oberen, und myxo-fibrös in den tieferen Lagen, mit zahlreichen Uebergängen von einer Gewebsform in die andere. Die Lymphgefässe sind überaus zahlreich, so zwar, dass sie das erwähnte honigwabenartige Aussehen bedingen, und man versucht wäre, streckenweise ron einem Lymph- 
angiom zu sprechen. Die Lymphgefässe sind vielfach buchtig, beträchtlich erweitert, hie und da zu Cysten ähnlichen Räumen umgewandelt, und erscheinen theils leer, theils mit Lymphkörperchen erfüllt, zwischen welchen ein zartes Netz von geronnenem Fibrin, und wechselnde Mengen von geronnenem feinkörnigen Eiweiss zu erkennen sind. Die Blutgefässe dagegen sind spärlich, grösstentheils stark erweiterte, mit Blutkörperchen erfüllte Capillaren. Die Endothelien sowohl der Blut- wie der Lymphgefässe sind ungewöhnlich gross.

Auffallend ist die grosse Menge von Bündeln glatter Muskelfasern. Eine wirkliche Neubildung derselben ist jedoch in hohem Grade unwahrscheinlich, vielmehr dürien wir nur an eine Bildungsanomalie denken. Auf eine solche Anomalie wird wohl auch die überraschende Menge von Lymphgefässen zurückzuführen sein. Die Muskelbündel befinden sich im Zustande einer interstitiellen Hyperplasie, das heisst das Bindegewebe zwischen den spindelförmigen Muskelfasern ist vermehrt, feinstreifig und reichlich mit kleinen Protoplasmakörpern versehen. Die Muskelspindeln selbst befinden sich vielfach im Zustande hydropischer Verquellung, welche das Protoplasma aufgehellt und dessen Körner auseinander geworfen hat. Die stäbchenförmigen Kerne sind von dieser Verquellung frei geblieben und sowohl im Längswie im Querschnitte deutlich erkennbar.

Die Maschenräume des myxomatösen, wie auch des myxofibrösen Gewebes sind vielfach von einer serösen, an Eiweiss reichen Fliissigkeit durchtränkt, welche sich an den in Chromsäure gehärteten Präparaten durch feine Körnung (geronnenes Eiweiss) kennzeichnet. Schon Virchow hat diese Form als eine congenitale bezeichnet. Wenn die Anschauung richtig ist, dass es sich hier um eine congenitale Vermehrung und Vergrösserung der Lymphgefässe handelt, dann können wir den Befund erklären, in dem wir sagen, dass in Folge ron Lymphangoitis wiederholte entzündliche Nachschübe im Derma aufgetreten sind, welche schliesslich zur Hyperplasie von vorwiegend myxomatösem Gewebe geführt hat. Da dieses Gewebe in einem frühen Entwicklungsstadium des Derma vorhanden ist, würde das mikroskopische Bild der elephantiatischen Schamlippe einem embryonalen Zustande entsprechen. 
Mikroskop. Untersuchung. über die chronische plastische Dermatitis. 199
Studiren wir nun ein Präparat von Elephantiasis dura. (Siehe Fig. 5.)

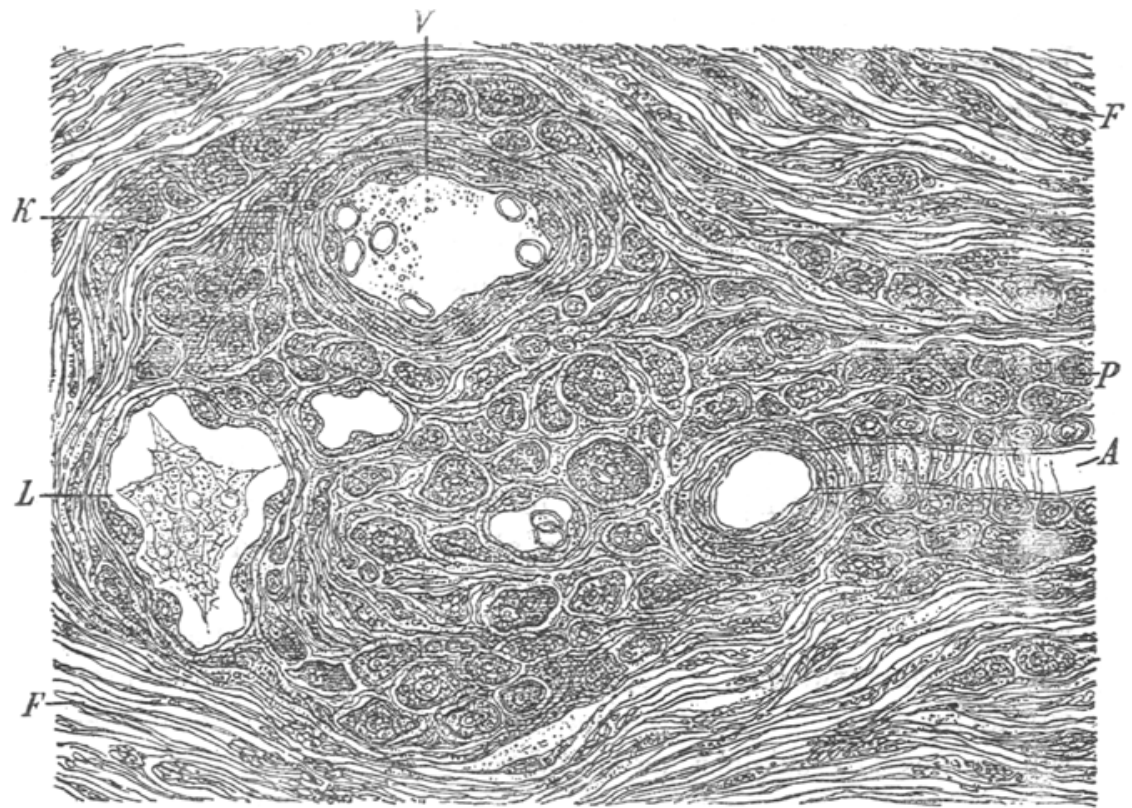

Fig. 5. Chronische plastische Dermatitis. Elephantiasis der grossen Schamlippe. Vergrösserung ooofach. $A=\Delta$ rteriole. $V=$ Vene. $L=$ Lymphgefässe. $P=$ Protoplasmakörper um die Arteriole. $K=$ Kette von Protoplasmakörpern. $F F^{\prime}=$ Fibröses Bindegewebe.

Bei dieser Form erscheint die Oberfläche dem freien Auge kleinhöckerig, die Hauptmasse nahezu solid, bei durchfallendem Lichte von seidenartigem Glanze. Mit dem Mikroskope erkennt man, dass die Epithellage von etwa normaler Breite, die Stachelschicht von der Hornschicht ohne Stratum granulosum und ohne Stratum lucidum getrennt ist. Die Hornschicht selbst ist schmal. Die Einsenkungen des Epithels sind nur seicht, entsprechend den seichten Papillen; von eigentlichen Epithelverlängerungen (Haare, Talg- und Schweissdrüsen) ist keine Spur $\mathrm{zu}$ erkennen. Die Hauptmasse des Gewebes besteht aus sogenanntem zarten fibrillären Bindegewebe, insbesondere zart im Papillarkörper und etwas gröber in der Hauptmasse des hyperplastischen Derma. Auch dieses Gewebe besteht aus sich durch- 
kreuzenden schmalen Bündeln, in deren Zwischenräumen vielfach kernhaltige Protoplasmakörper liegen.

Bündel von glatten Muskelfasern sind nur spärlich vorhanden; die Lymphgefässe in normaler Menge oder zum Mindesten nur wenig vermehrt, jedoch in ausgesprochener Weise erweitert. Man erkennt dieselben unter dem Mikroskope an den grossen Endothelien und der, in der Regel geschrumpften Füllmasse, aus coagulirtem Fibrin, Eiweiss und spärlichen Lymphkörperchen bestehend. Blutgefässe sind zahlreich, insbesondere Arteriolen in überwiegender Menge vorhanden. Die Venen haben gleichfalls eine deutliche Muskelschichte. Die Capillargefässe sind nicht erweitert.

Den auffälligsten Befund bilden zahlreiche Nester von Entzündungskörpern, entweder in Begleitung von Blut- und Lymphgefässen oder von Arteriolen allein. Die Entzündungsnester sind nämlich am zahlreichsten längs den Arteriolen, und nicht, wie man der herrschenden Anschauung über Lymphangoitis entsprechend erwarten sollte, längs der Lymphgefässe. Betrachten wir uns ein solches Entzündungsnest bei mittleren Vergrösserungen des Mikroskopes, so erkennen wir um jedes grobkörnige oder nahezu homogene Entzündungskörperchen herum ein zartes, myxomatöses Bindegewebsnetz. Das entzündete Gewebe befindet sich demnach in einem frühen myxomatösen Stadium, aus welchem dasselbe den entwicklungsgeschichtlichen Vorgängen gemäss in das Stadium des fibrösen übertritt und zur Vermehrung des letzteren führt. In der Nähe der Entzündungsnester begegnen wir häufig kettenförmigen Protoplasmakörpern zwischen den Bindegewebsbündeln, über deren Herkunft ich schon in der Arbeit über acute Dermatitis Aussagen gemacht. Für eine Annahme einer Auswanderung farbloser Blutkörperchen im Sinne Cobnheims finden wir nirgends Anhaltspunkte.

Die Ergebnisse meiner Untersuchungen über plastische Dermatitis lassen sich in folgenden Punkten zlsammenfassen:

1. Nach Aufhören der acuten Entzündung erfolgt eine Rückkehr der Klümpchen neugebildeter lebender Materie in das Stadium indifferenter Protoplasmakörper.

2. Im Stadium der Indifferenz werden nicht nur das Proto- 
Mikroskop. Untersuchung. über die chronische plastische Dermatitis. 701

plasma, sondern auch die Kerne reticulirt und letztere hören damit auf, als solche erkennbar zu sein.

3. Die Mascheuräume des Protoplasmas sind ursprünglich mit einer stickstoffhaltigen Flüssigkeit erfüllt. Diese Flüssigkeit wird $\mathrm{zu}$ einer mehr soliden stickstoffhaltigen, leimgebenden Grundsubstanz umgewandelt; während das Netz der lebenden Materie zwar erhalten bleibt, jedoch ohne Anwendung von Reagentien nicht mehr sichtbar ist.

4. Erfolgt die Infiltration mit Grundsubstanz direct in den indifferenten Protoplasmakörpern, dann entstehen neugebildete Bündel von Bindegewebe, ohne Streifung. Zerfallen jedoch die Protoplasmakörper vor der Infiltration zu zarten Spindeln. dann erfolgt die Bildung von Bündeln streifigen Bindegewebes.

5. In beiden Fällen bleiben unveränderte Zuige des Protoplasmas zwischen den neugebildeten Bindegewebsbündeln, ebenso wie im normalen Derma.

6. Bei manchen Formen der hyperplastischen Dermatitis entsteht anstatt fibrösen, ein myxomatöses Bindegewebe. welches einem Stillstande in der Entwicklung des Derma entsprechend dem dritten bis vierten Embryonal-Monate zukommt. 